\title{
6 Verknüpfungen zwischen den dispositiven Elementen
}

Ein Dispositiv kann erst dann als Dispositiv, d.h. als eine wirkmächtige Regierungstechnologie beschrieben werden, wenn die Elemente, aus denen es bestehen soll, aufeinander verweisen und miteinander verknüpft sind. Die bisherigen Teilanalysen zu den jeweiligen Elementen haben die Verknüpfungen und Verhältnisbestimmungen "zwischen Diskurs, Nicht-Diskurs, Subjektivation und Objektivation in Bezug auf sozialen Wandel« (Bührmann \& Schneider 2012: 109) bereits angedeutet.

Das Ziel dieses Kapitels besteht darin, die bestehenden Verhältnisbestimmungen noch stärker zu verdeutlichen und die wechselseitigen Verbindungen zwischen den Elementen des Präventionsdispositivs anschaulich zu rekonstruieren. Dabei sollen sie nicht als per se vorausgesetzt oder zwingend in Beziehung zueinander gebracht werden. Vielmehr richtet sich die Rekonstruktion ihrer Verhältnisse darauf, ihre gegenseitige Bedingtheit und Angewiesenheit, und damit ihre Zugehörigkeit und Funktionalität, zu überprüfen. Im Folgenden werden also die Verhältnisbestimmungen zwischen Diskurs und nicht-diskursiven Praktiken, zwischen Diskurs und Objektivationen, zwischen Diskurs und Subjektivationen, sowie zwischen Subjektivationen und nicht-diskursiven Praktiken, zwischen Subjektivationen und Objektivationen, sowie zwischen nicht-diskursiven Praktiken und Objektivationen beschrieben (s. Abb. 1).

\subsection{Diskurs und nicht-diskursive Praktiken}

Der Diskurs wurde im Rahmen dieser Untersuchung auf drei Ebenen untersucht als Spezialdiskurs, als Interdiskurs und als Elementardiskurs -, die das Wissen um Prävention in unterschiedlichen Ausprägungen - als Aufforderung zum Arbeitsschutz (Spezialdiskurs), als Teil der Unternehmenskultur (Interdiskurs), als ein Weg zurück in die Normalität (Elementardiskurs) - produzieren und transferieren. Die Analysen konnten zeigen, wie das Wissen in diesem Diskurs zirkuliert und verschiedene Formen annimmt.

Demgegenüber wurden die nicht-diskursiven Praktiken daraufhin untersucht, wie sich das Wissen um die Prävention des Burnout-Syndroms in konkrete Handlungen und Verhaltensweisen umsetzt. Am Beispiel von konkreten Umsetzungsweisen (ErgoScouts, Gamification u.a.) wurde gezeigt, wie sich Prävention auf natürliche Weise in den (Unternehmens-)Alltag integriert und als eine nicht-diskursive, nicht sprachlich artikulierte Praxis vollzogen hat.

Illustrierend für die Verknüpfung dieser beiden Dispositivelemente kann die Praxis der interessierten Selbstgefährdung dienen (nicht-diskursive Praktiken), die in den Geständnissen von Burnout-Betroffenen (Elementardiskus) in gleicher Weise vorkommt. So berichten viele von ihnen, dass sie trotz der Gefährdungen nicht 
imstande waren, ihr hohes Leistungstempo zu reduzieren (vgl. Yvette 2008: 18; Silvia 2009: 15; Roland 2012: $52 \mathrm{f}$ u.a.). Genau auf diese Art und Weise wird die interessierte Selbstgefährdung auch von den Arbeitsorganisationsforschern beschrieben (Interdiskurs), die sie dann in ihre Empfehlungen zur Verbesserung des innenbetrieblichen Gesundheitsmanagements miteinbeziehen. Interessierte Selbstgefährdung tritt dann nicht als betriebliche Störung auf, sondern als persönliche Unfähigkeit, die die Unternehmen wahrnehmen und für die Erreichung ihrer Ziele einsetzen können. Somit verbindet diese Praxis den Diskurs mit den nicht-diskursiven Praktiken.

\subsection{Diskurs und Objektivationen}

Der Diskurs um Prävention vollzieht sich nicht nur in nicht-diskursiven Praktiken, sondern auch in Gegenständen und Objekten. Diese Vergegenständlichungen bzw. Objektivationen von diskursivem Wissen wurden anhand verschiedener Kleingeräte wie Wearables oder Bioresonanzgeräte beschrieben, aber auch als räumliche ergonomische Einrichtungen oder ganze architektonische Konzepte wie das der heilenden Architektur.

Ein konkretes Beispiel, wie sich die Objektivationen soz. zurück auf die Diskurse beziehen, zeigt die Integration der Wearables-Apps (Objektivationen) in das betriebliche Gesundheitsmanagement (Interdiskurs). Hier werden vor allem die Führungskräfte als Zielgruppe angesprochen, da sie vor zahlreichen Aufgaben der Selbstsorge und der Sorge um die Beschäftigten stehen. »Die App >Gesund führen hilft Führungskräften, diese Herausforderungen zu meistern, psychische Überlastungen bei Mitarbeiterinnen und Mitarbeitern zu erkennen und rechtzeitig gegenzusteuern - denn psychische Gesundheit ist Führungsaufgabe! « (psyGa 2013 [Herv. i. O.])

Ein zweites Beispiel bietet die Anwendung der HRV- oder Bioresonanzmessgeräte (Objektivationen), von denen ein konkreter Gebrauch in den Unternehmen (Interdiskurs) sowie bei den Burnout-Betroffenen (Elementardiskurs) gemacht wird. So bieten die HRV-Messgeräte die Messung der Schlafqualität der Beschäftigten, womit sie aus dem Schlaf »eine strategische Ressource und de[n] Schlüssel zur nachhaltigen Erhaltung der persönlichen Leistungsfähigkeit «machen (Ebner 2018: 200). Gleichzeitig werden die Bioresonanzgeräte in der individuellen Therapie als Präventionsmittel eingesetzt, wie dies ein Burnout-Betroffener beschrieben hat (vgl. Thomas 2006: 66).

Auch weitere Themen durchkreuzen und verbinden die Objektivationen mit dem Diskurs, wie die Sorge um den Körper und die dafür zur Verfügung stehenden präventiven Vergegenständlichungen einer heilenden Architektur. Damit wird nachgewiesen, wie diese beiden Elemente verbunden sind und sich gegenseitig, bspw. durch neue technologische Fortschritte und Möglichkeiten, bedingen. 


\subsection{Diskurs und Subjektivationen}

Alle drei Diskursebenen haben auf eine bestimmte Art und Weise unterschiedliche Formen der Subjektivierung produziert, die sich zum Teil ergänzen, zum Teil überlappen und zum Teil weiterentwickelt haben. Dabei ist zu betonen, dass dies keineswegs in zeitlicher Abfolge erfolgt.

So hat der Spezialdiskurs mit der Aufforderung zum Arbeitsschutz und zur Prävention am Arbeitsort ein Subjekt hervorgebracht, das gefährdet ist und daher geschützt werden soll. Deswegen müssen nicht nur internationale Organisationen, sondern auch staatliche Regierungen und Verbände die Aufgabe übernehmen, entsprechende Leitlinien zu entwickeln und im Rahmen des Arbeitsschutzes das Interesse an der Prävention durchsetzen.

Der Interdiskurs hat die Wechselbeziehungen zwischen den Beschäftigten, den Führungskräften und dem Aufsichtspersonal bzw. dem betrieblichen Gesundheitsmanagement problematisiert und den jeweiligen Akteursgruppen im Unternehmen unterschiedliche präventive Subjektmodi zugeteilt. Demnach figurieren die Führungskräfte als Vorbild und gleichzeitig selbst als bedrohte Subjekte und müssen diese doppelte Aufgabe der Selbstsorge und der Sorge um die anderen meistern. Die Beschäftigten spielen eine besondere Rolle in der Unternehmensprävention, indem sie als Experten in eigener Sache auftreten und daher die Entscheidung über den Unternehmenserfolg bzw. - misserfolg in eigenen Händen haben. Das Aufsichtspersonal wird demgegenüber vor die Aufgabe gestellt, die selbst-gefährdenden Subjekte, seien es nun Führungskräfte oder Beschäftigte, in einen auf ein Minimum reduzierten Gefährdungsmodus zu bringen.

In dem Elementardiskurs haben sich die Betroffenen mit sich selbst und mit ihrer Burnout-Störung auseinandergesetzt und verschiedene Facetten des ausgebrannten Subjekts produziert. Zu diesen zählen vor allem die Übernahme der Selbstverantwortung für das Burnout sowie die darauffolgende Therapie und zukünftige Prävention. Die Betroffenen müssen lernen, mit der Burnout-Erfahrung und mit der eigenen Labilität umzugehen, um den möglichen Wiederausbruch zu verhindern.

Die verschiedenen Verschränkungen zwischen dem Diskurs und der Subjektivation zeigen, wie sich diese Elemente gegenseitig bedingen, hervorbringen und wieder aufeinander beziehen.

\subsection{Subjektivationen und nicht-diskursive Praktiken}

Die Verschränkung der Subjektivation mit den nicht-diskursiven Praktiken wurde vor allem in der Schreibpraxis sichtbar. Indem die Burnout-Betroffenen in ihrer Suche nach Verständnis der eigenen Situation eine Praxis des Selbsterkennens als Schreibpraxis betrieben haben, haben sie sich zu sich selbst in unterschiedlicher 
Weise positioniert und verschiedene Subjektmodi entwickelt. Das Stöbern in sich selbst, die Auseinandersetzung mit der eigenen Vergangenheit, die Qual der Sorge um die eigene Gesundheit, um die Zukunft und die Sicherung des eigenen Wohlstands, die Konflikte mit den Arbeitgebern und Familienmitgliedern oder Behörden, die Geständnisse vor sich selbst und vor den Therapeuten - all das haben sie schriftlich verfasst und als eine Projektionsfläche dargestellt, damit sie die innere Landschaft in sich selbst verbildlichen und anschauen konnten. Damit wurde die Beziehung zu sich selbst und die Beziehung zu den anderen, und mithin auch das Subjektverständnis von sich selbst, neu austariert.

Ein verbindendes Glied der nicht-diskursiven Praktiken und der Subjektivationen stellen auch die Praxis der interessierten Gefährdung, die oben beschrieben wurde, oder auch die einfachen Praktiken der Sorge um den Körper, um die Emotionen oder um das Denken. Sport treiben, sich gesunder ernähren, langsamer und bewusster atmen stellen Praktiken dar, die sich rückwirkend auf die Herstellung eines ausbalancierten, bewusst-lebenden und leistungsfähigen Subjekts beziehen.

Die nicht-diskursiven Praktiken und die Subjektivation verschränken sich an mehreren Stellen, auch dort, wo die Praktiken nicht als Präventionsmaßnahmen entstanden sind - wie gesundes Essen, Schlafen oder Sport - , sondern unter ihre Rationalität sukzessive miteinbezogen wurden.

\subsection{Subjektivationen und Objektivationen}

Auch Subjektivationen und Objektivationen als dispositive Elemente verbinden und unterstützen sich gegenseitig. Diese Verschränkung hat eine viel ältere Geschichte und geht auf die moderne wissenschaftliche Entdeckung des Menschen und seines Organismus, seiner psychischen Zustände und seines Körpers zurück. Zu der Zeit, wo der Mensch zum Objekt der Wissenschaften und der Wissenserforschung geworden ist, haben sich auch seine Untersuchungsmethoden und-instrumente entwickelt und etabliert. Diese gegenseitige Verschränkung von Technologien und Menschenlehre lässt sich auch an den gegenwärtigen Instrumenten beobachten.

Ein konkretes Beispiel bieten die Fitness-Tracker, die an dem Körper des Subjekts getragen werden und ihn über seinen gesundheitlichen Zustand taktmäßig informieren. Das Subjekt hat damit das Wissen darüber, welche Abläufe in seinem Körper gerade passieren und was es als nächstes zu tun hat. Dank ihrer Hilfe wird der Körper des Subjekts zu seinem Objekt, zu einem objektivierbaren und messbaren Gegenstand. Sodann kann ihn die Präventionsrationalität als das richtige Präventionsinstrument umdeuten und das Subjekt als jenes stilisieren, das seine Gesundheit und Leistungsfähigkeit in den eigenen Händen hat.

Ähnlich dazu verhält sich auch die Kultur der heilenden Architektur, die die Subjekte anspricht, die um ihre Gesundheit Sorge tragen und Orte suchen, wo sie 
wieder neu auftanken und weiterleben können. Die unterschiedlichen Ausprägungen der Büroarchitektur zeigen zudem, wie sich das Subjekt in einem wachen $\mathrm{Zu-}$ stand halten, seinen Kraftverlust reduzieren und seine Leistungen dosieren kann. Somit wirkt sich das materielle Milieu auf das Subjekt in einer Weise aus, die seine freiwillige präventive Selbstsorge voraussetzt.

Die Objektivationen und die Subjektivationen verschränken sich kontinuierlich, indem die neuen Geräte und architektonischen Einrichtungen einen Subjektmodus hervorbringen und sich an ihn und seine Beziehung $\mathrm{zu}$ sich selbst fortlaufend anpassen. Die neoliberale Sorge um sich bekräftigt dieses gegenseitige Verhältnis, indem sie das Subjekt in ständiger Gefährdung vor dem Verlust der Gesundheit - und damit der Leistungskraft und des Wohlstands - festhält.

\subsection{Objektivationen und nicht-diskursive Praktiken}

Auch Objektivationen und nicht-diskursive Praktiken verbinden sich und bedingen sich gegenseitig. Die neu hervorgebrachten Instrumente zur präventiven Selbstsorge werden mit konkreten Gebrauchsanweisungen verbunden, die die burnoutbetroffenen oder gefährdeten Subjekte befolgen müssen.

So erfordern die Gesundheits-Apps bestimmte physische Aktivitäten, damit die relevanten Informationen überhaupt erst aufgenommen werden können. Nach ihrer Auswertung folgen dann Empfehlungen zur Verbesserung der Aktivität oder ihrer Anpassung an das gewünschte Ergebnis. Das Laufen kann somit zu einem präventiven Laufen, das kurze Pausieren zu einem Aufatmen im Arbeitsmarathon und die achtsamen Essgewohnheiten zu stressreduzierenden Maßnahmen werden.

Auch die Ergo-Geräte fordern eine gesundere und präventivere Arbeitsweise, indem sie die bisherigen Handlungen ergonomischer und der natürlichen Körperhaltung und -bewegung angepasster machen und damit zu mehr Entlastung, Effektivität und zum besseren Wohlbefinden führen. Schließlich erzielen präventiv gestaltete Räumlichkeiten und architektonische Arrangements einen veränderten Umgang mit sich selbst. So fordert, z.B. das Konzept der Reversible Destiny Lofts eine gezielte Anstrengung, die »vor allem bei älteren Menschen dem geistigen und körperlichen Niedergang entgegenwirken« soll (Allplan Deutschland $\mathrm{GmbH}$ 2018).

Die nicht-diskursiven Praktiken und Objektivationen stehen in enger Verbindung zueinander, indem die technologischen Entwicklungen zu veränderten Handlungen und Umgangsweisen führen.

\subsection{Zwischenfazit}

Wie die knappen Darstellungen der Dispositivelemente nahegelegt haben, bestehen diese durchaus in einer engen Verbindung zueinander und bestätigen somit 
die These, dass das Dispositiv der Prävention zurecht als eine Machtstrategie, wie sie Michel Foucault bezeichnet hat, betrachtet werden kann.

Außerdem, indem sich die dispositiven Elemente aufeinander beziehen, bleiben sie als ein funktionierendes Oberflächennetz »in einigen großen Wissens- und Machtstrategien miteinander« verkettet (Foucault [1976] 1977: 128). Eine Wissensund Machtstrategie wie das Präventionsdispositiv ist zudem in soziale und gesellschaftliche Entwicklungen und Wandlungen eingebettet, die seine Funktionsweise bestimmen und die Möglichkeiten seiner Machtauswirkungen vorschreiben. Demnach kann sich dieses Dispositiv der Elemente anderer Dispositive bedienen, diese umwandeln und für eigene Zwecke nutzbar machen, wie dies mit den Elementen des Sicherheitsdispositivs oder des Aktivierungsdispositivs geschehen ist. Das gleiche gilt auch umgekehrt.

Das Dispositiv der Prävention kann schließlich durch seine Elemente unterschiedliche Themenfelder aufgreifen - wie das betriebliche Gesundheitsmanagement, die Krankenpflege, die soziale Vorsorge usw. - und diese als Zielscheibe der Politik (re-)definieren. Wenn es dann konkret um die Prävention von BurnoutSyndrom geht, können sich die Methoden durchaus auf andere psychische Störungen und Zivilisationskrankheiten ausdehnen - Depression, Histrionie, Borderline, ADHS, Boreout-Syndrom, Schizophrenie, Angststörungen usw. - und durch deren Problematisierung weitere Kenntnisse erhalten. Mit der funktionierenden Verbindung seiner Elemente wirkt das Dispositiv als ein Oberflächennetz quer durch die gesellschaftlichen Diskurse und sichert dadurch sein Überleben und Fortschreiben. 
